\title{
Using AC Conductivity Measurements to Study the Influence of Mechanical Stress on the Strength of Geomaterials
}

\author{
Ilias Stavrakas ${ }^{1}$, Kostantinos Moutzouris ${ }^{1}$, Kostantinos Ninos ${ }^{1}$, Nikos Mitritsakis ${ }^{2}$, \\ Zacharias Agioutantis ${ }^{2}$, Dimos Triantis ${ }^{1}$ \\ ${ }^{1}$ Department of Electronics, Technological Educational Institute of Athens, Athens, Greece \\ ${ }^{2}$ Department of Mineral Resources Engineering, Technical University of Crete, Chania, Greece \\ Email: ilias@ee.teiath.gr
}

Received March 6, 2012; revised April 10, 2012; accepted April 20, 2012

\begin{abstract}
The Dielectric Spectroscopy technique is a tool that can be used to provide information regarding the physical and chemical properties of materials. In this work Dielectric Spectroscopy (DS) measurements were conducted on marble specimens that were previously subjected to uniaxial compressive stress up to fracture in order to investigate the influence of the mechanical stress on the dielectric properties of the specimens. Specifically, the ac conductivity $\left(\sigma_{\mathrm{ac}}\right)$ was measured when an ac electric field in the frequency range $1 \mathrm{kHz}-1 \mathrm{MHz}$ was applied upon dry and saturated specimens which were subjected successively to higher levels of mechanical stress. The experimental results indicate that there are systematic variations in the values of the ac conductivity after each stress application at a higher stress level. Such variations become more intense at higher stress values and can be used to indicate the upcoming fracture since significant increase of conductivity is recorded when microcracks formations appear and propagate in the sample bulk.
\end{abstract}

Keywords: AC Conductivity Measurements; Marble; Mechanical Load

\section{Introduction}

Mechanical stress applied on rocks creates microscopic and macroscopic discontinuities resulting in changes in their mechanical behaviour [1-3]. Marble is a metamorphic rock with imperfections in its structure due to either internal or external factors such as mechanical strain, chemical or physical processing which play an important role in the behaviour of the material.

Various laboratory experiments on marble samples have been recently conducted in order to determine the impact of the mechanical stress and the consequent mechanical damage on the electrical properties of geomaterials. Such experiments include Dielectric Spectroscopy in the frequency domain $[4,5]$ and Isothermal Depolarization Currents [6].

Dielectric properties are related to the capability of a material to be polarised under the influence of an externally applied electrical field. The polarisability of a material depends on its structure and molecular properties and therefore dielectric measurements can provide information in this respect. Dielectric Spectroscopy (DS) is an electrical technique that has been used to characterize the rock microstructure and to provide useful information about the relationships governing microstructure, elec- trical properties, and chemical processes during hydration [7-9]. The complex relative permittivity $\varepsilon^{*}$ (hereafter, referred to as complex permittivity for convenience) is defined as:

$$
\varepsilon^{*}=\varepsilon^{\prime}-j \varepsilon^{\prime \prime}=\varepsilon^{\prime}+\left(\sigma / j \varepsilon_{0} \omega\right)
$$

where $\varepsilon^{\prime}$ and $\varepsilon^{\prime \prime}$ are the real and imaginary parts of $\varepsilon^{*}$ respectively, $\sigma$ is the electrical conductivity, $\varepsilon_{0}$ is the vacuum permittivity and $\omega$ is the angular frequency $(\omega=2 \pi f)$. It is widely observed that the ac conductivity with respect to frequency obeys a power law $[10,11]$. Jonscher [10] suggested that this law constitutes a "universal" property of the materials. In general, ac conductivity $\sigma(f)$ is found to vary with frequency $\mathrm{f}$ as:

$$
\sigma(f)=\sigma(0)+A \cdot f^{n}
$$

at frequencies well below the lattice vibrational frequency [10]. In Equation (2), $\sigma(0)$ is the dc limit of conductivity, $A$ is a parameter depending on temperature and pressure and the exponent $n$ takes values between 0 and 1. In the high frequency range since $A \cdot f^{n} \gg \sigma(0)$ Equation (2) can be written as follows:

$$
\sigma(f)=A \cdot f^{n}
$$


In this work, marble specimens were subjected to uniaxial compressive stress in order to cause damage to their bulk. After each stress application and while the specimen remained unstressed two sets of ac conductivity measurements were conducted in the frequency range between $1 \mathrm{kHz}$ and $1 \mathrm{MHz}$. During the first set of measurements the specimen included its natural moisture while during the second set the natural moisture of the specimen was removed after heating. Initially, pilot ac conductivity measurements were conducted for all the specimens used.

\section{Materials and Experimental Techniques}

\subsection{Materials}

Marble belongs to the class of metamorphic rocks. Its structural inhomogeneities are due to either natural or man-made causes like the application of mechanical or chemical processing. The marble specimens used were prepared from samples collected from Mt. Penteli, Attica, Greece. Marble from this area is mainly composed of calcite (98\%) and other minerals, such as muscovite, sericite and chlorite. Its content in quartz is very low $(0.2 \%)$, while its density is $2.7 \mathrm{~g} / \mathrm{cm}^{3}$ and its porosity is approximately $0.4 \%$. Matrix rocks of the above origin were intentionally selected to be quasi single grained. The dimensions of the prismatic specimens subjected to compression were $38 \mathrm{~mm} \times 20 \mathrm{~mm} \times 9 \mathrm{~mm}$. The mechanical stress was applied in parallel to the long edge of the specimens.

The specimens were cleaved from the rock using two different directions (see Figure 1). According to the direction of cleavage the specimens were named as type-1 and type-2. The dashed lines of Figure 1 correspond to the chlorite $\left((\mathrm{Mg}, \mathrm{Fe}, \mathrm{Al})_{6}(\mathrm{Si}, \mathrm{Al})_{4} \mathrm{O}_{10}(\mathrm{OH})_{8}\right)$ and muscovite $\left(\mathrm{KAl}_{2}\left(\mathrm{AlSi}_{3}\right) \mathrm{O}_{10}(\mathrm{OH})_{2}\right)$ layers of the material.

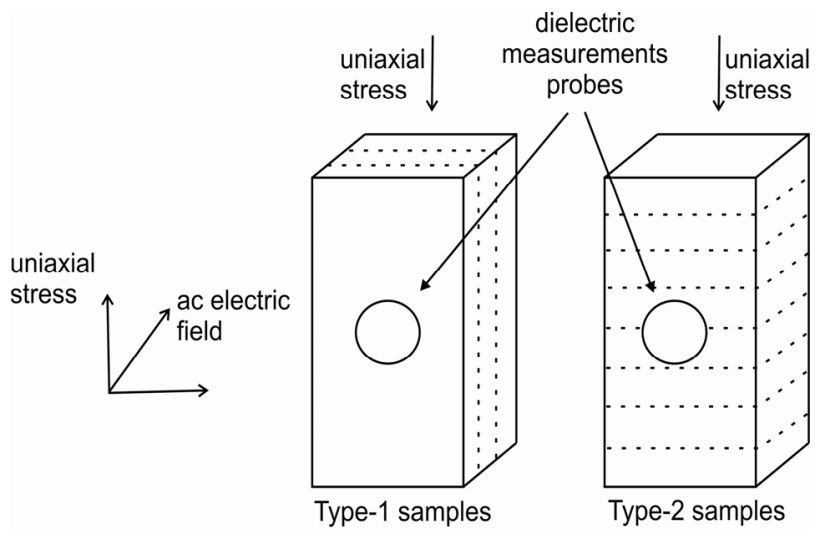

Figure 1. Orientation of the specimens according to the directions of the applied stress, the dielectric measurement probes and the conductive layers of muscovite and chlorite (type-1 and type-2 specimens).
It must also be noted that these layers due to their chemical compositions are considered to be more conductive than calcite which is the main mineral in marble. Both types of specimens were tested in the subsequent experiments.In order to achieve acceptably dry material, the specimens were heated for 24 hours in a constant temperature chamber at $378 \mathrm{~K}$. The failure load of the samples was detected after preliminary tests to vary between $8.6 \mathrm{kN}$ and $10 \mathrm{kN}$ for both types of specimens.

\subsection{Experimental Technique}

The experimental apparatus that was used to conduct the uniaxial compression test on the specimens is shown in Figure 2 and comprises a stiff loading frame (MTS-815). In this apparatus, the spherical seat is incorporated in the upper loading platen of the frame. The load force was parallel to the longest side of the specimen. The load on the specimens was applied continuously at a displacement rate equal to $0.001 \mathrm{~mm} / \mathrm{s}$. After reaching the predetermined stress level, the load was removed suddenly.

The selected mechanical load levels applied on the specimens were $3.2 \mathrm{kN}, 3.8 \mathrm{kN}, 4.2 \mathrm{kN}, 6.0 \mathrm{kN} 7.3 \mathrm{kN}$ and $8.5 \mathrm{kN}$. After each loading ac conductivity measurements were conducted. The experimental arrangement for conducting the ac conductivity measurements (see Figure 3) comprises an LCR meter (Agilent model 4284A), accompanied by a dielectric test fixture (Agilent model 16451B) and further supported by a computer for data recording, storage and analysis. The dielectric test fixture that was used to hold the specimen was protected by a cabin providing constant temperature $(298 \mathrm{~K})$, inert atmosphere and also low humidity by continuous effusion of nitrogen.

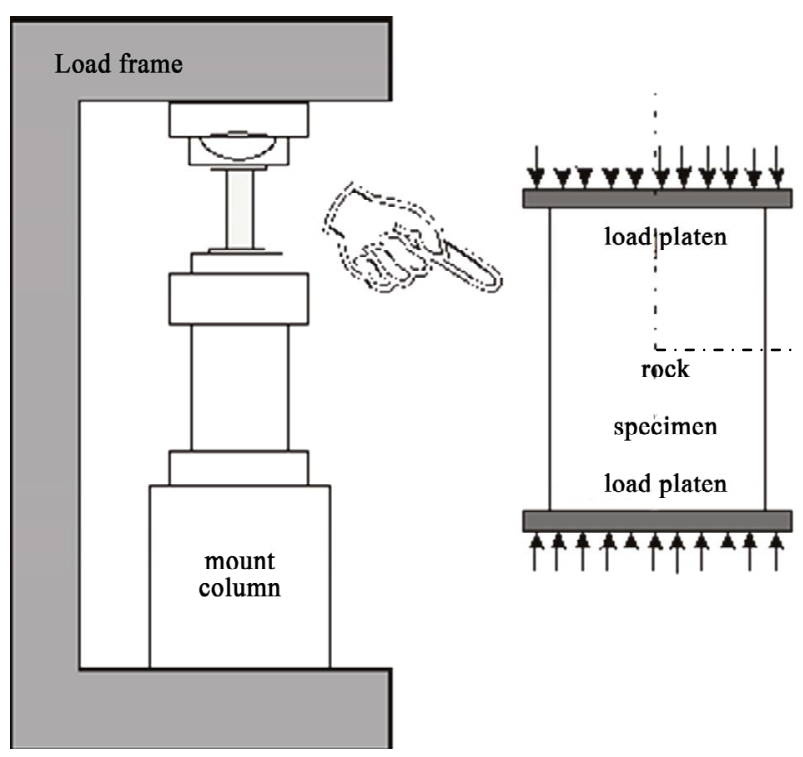

Figure 2. Typical testing system for uniaxial compression testing. 


\section{Results and Discussion}

Pilot ac conductivity measurements in the frequency range $1 \mathrm{kHz}-1 \mathrm{MHz}$ were conducted for type-1 and type-2 specimens without removing their natural moisture. Figure 4 shows the behaviour of the ac conductivity with respect to the frequency for type- 1 and type- 2 specimens. It becomes clear that type 2 specimens exhibit higher ac conductivity in the whole frequency range. This behaviour can be attributed to the fact that the external electric field applied to measure the ac conductivity was directed in parallel to the muscovite and chlorite layers that act as conductive paths between the two electrodes of the dielectric measurements probe (see Figure 1).

The high frequency part of the ac conductivity measurements was fitted with the universal conductivity power law (i.e. Equation 3). The fitting provided approximate values 0.64 and 0.80 for the exponential factor $n$ for type-1 and type- 2 specimens respectively. These values seem to be characteristic for the two types since the error deviation is very low (i.e. $0.64 \pm 0.03$ and $0.80 \pm 0.04$ respectively).

Figure 5 shows two representative ac conductivity experimental datasets with respect to frequency for type-1 specimens. Figure 5(a), shows the behaviour of the ac conductivity when the specimen without subjecting it to any mechanical load while $b$ shows the corresponding behaviour of the same specimen after applying a mechanical load of $7.3 \mathrm{kN}$.

As can be seen in the Figure 5 the applied load has no impact on the ac conductivity behaviour at the high frequency ranges $(>250 \mathrm{kHz})$ but it severely influences the ac conductivity values at the low frequencies. It becomes clear that the values of the ac conductivity become higher after applying the mechanical load on the specimen. This can be attributed to crack formation and propagation that create additional conductive paths at the crack edges.

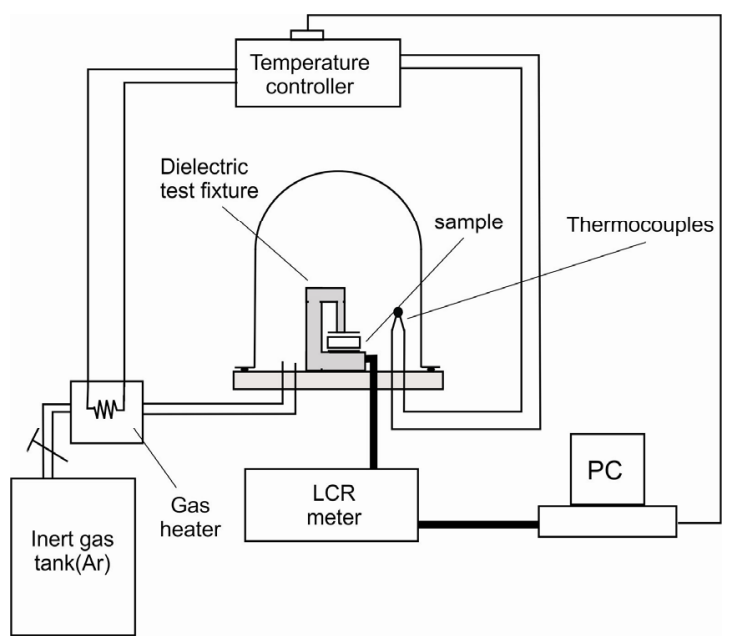

Figure 3. Dielectric measurements arrangement including the inert gas tank.

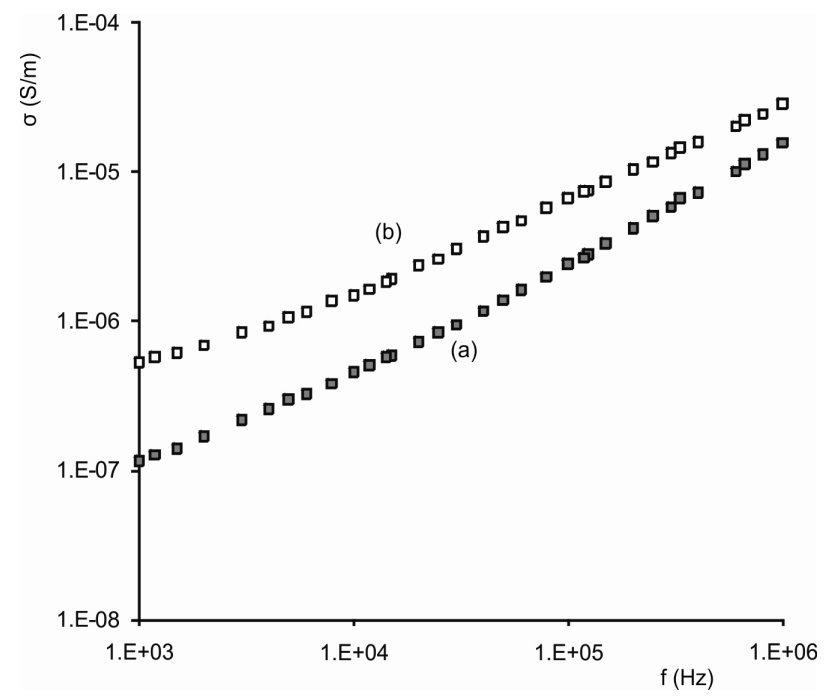

Figure 4. A typical set of experimental datasets demonstrating the behaviour of the ac conductivity with respect to frequency for both (solid squares, a) type-1 and (open squares, b) type-2 specimens.

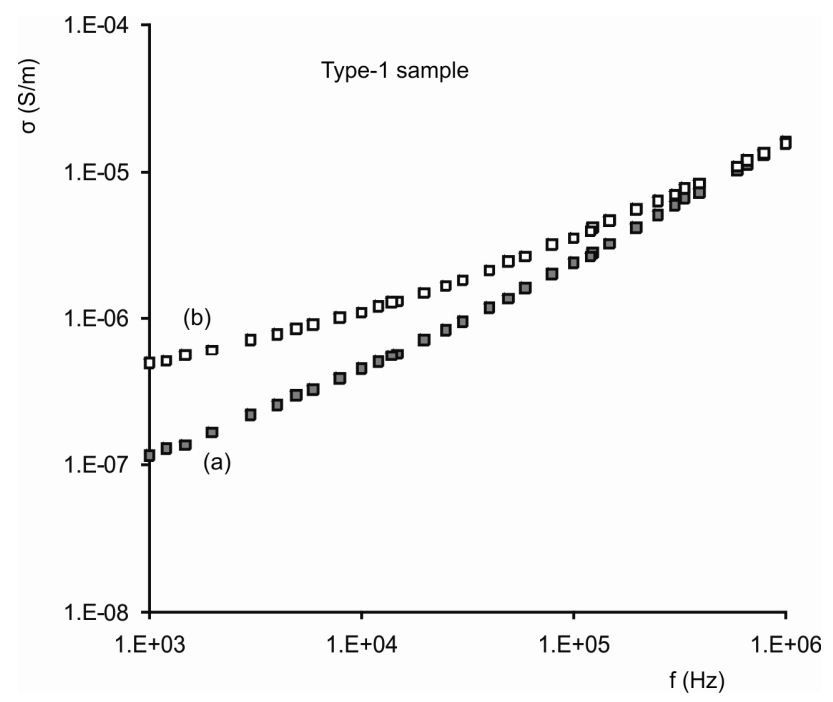

Figure 5. A typical set of experimental datasets demonstrating the behaviour of the ac conductivity with respect to frequency for type-1 specimen (solid squares, a) before and (open squares, b) after applying $7.3 \mathrm{kN}$ mechanical load.

This phenomenon provides greater freedom of charge motion and consequently higher ac conductivity in the low frequency range. The higher the frequency of the applied ac field the lower is the current flowing along the microcrack and macrocrack edges. The same behaviour is observed for the type-2 specimens (see representative datasets in Figure 6). Figure 6 clearly shows a variation of the values of the ac conductivity before (a) and after (b) applying mechanical load. Specifically, this variation becomes evident only in the frequencies below $120 \mathrm{kHz}$. The ac conductivity measurements of type- 1 specimens that had previously been loaded at $7.3 \mathrm{kN}$ was also fitted 
by the conductivity universal power law and factor $n$ found to be approximately equal to 0.63 . This reduction of the factor $n$ indicates that the specimen has been subjected to compressive stress [12]. Similar results were extracted for type 2 specimens (see Figure 6).

When the natural moisture of the specimens is removed by heating, the ac conductivity becomes lower. This is evident in Figure 7 which shows the behaviour of the ac conductivity with respect to frequency for a typical type-1 specimen before and after the application of mechanical load.

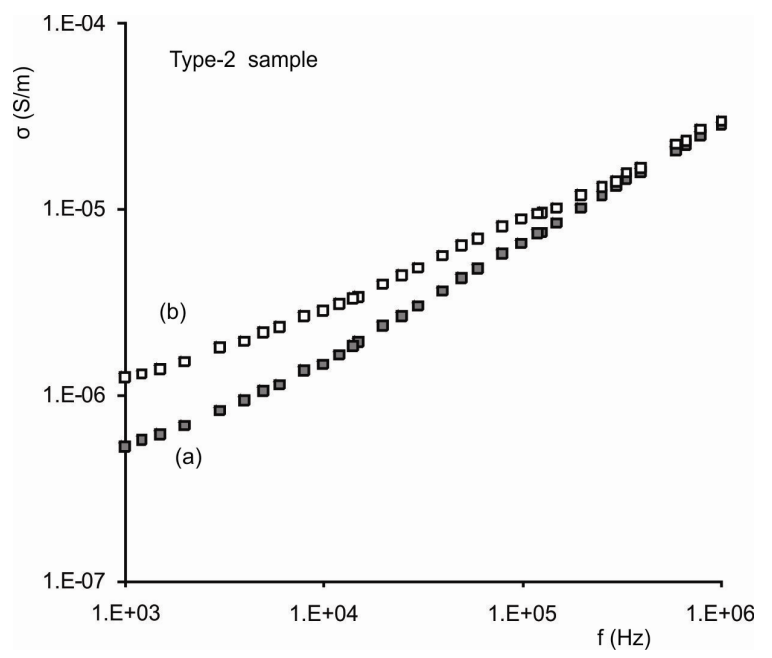

Figure 6. A typical set of experimental datasets demonstrating the behaviour of the ac conductivity with respect to frequency for type-2 specimen (solid squares, a) before and (open squares, b) after applying $8.5 \mathrm{kN}$ mechanical load.

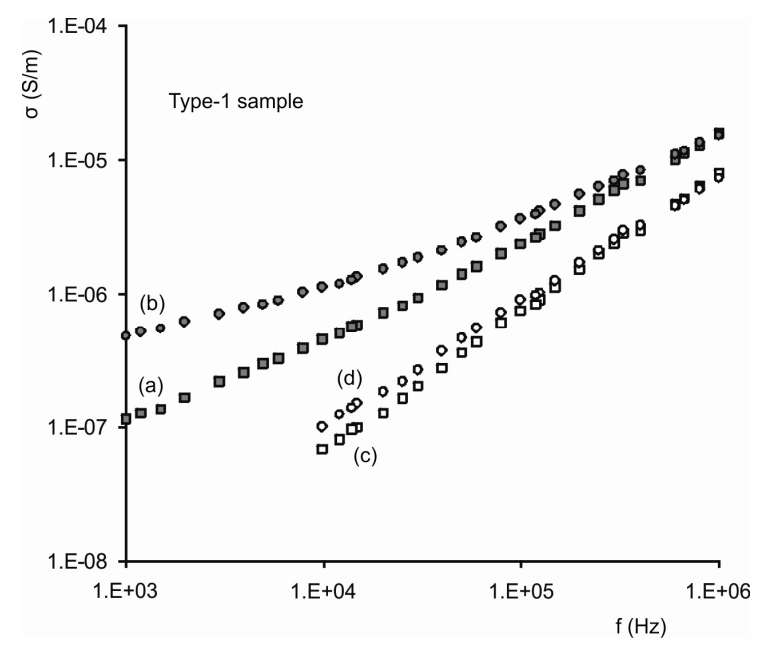

Figure 7. Representative experimental datasets of the ac conductivity in the frequency range between $1 \mathrm{k} \mathrm{Hz}-1 \mathrm{MHz}$ for type-1 specimen. (a) and (b) correspond to the specimens that maintained their natural moisture before and after applying mechanical load $(7.3 \mathrm{kN})$ respectively. (c) and (d) correspond to the specimens after removing their natural moisture before and after applying mechanical load respectively.
As it was expected when removing the natural moisture of the specimens, their conductivity decreased due to lack of free charges provided by water in the specimen system. The variation breadth of the ac conductivity due to the applied mechanical load is more intense for the specimens containing their natural moisture compared to the variation breadth for the dried specimens.

For the dried specimens a low frequency limit was set to the measurements due to conductivity values that become too low to comply with the measuring system specifications and the corresponding conductivity values exhibit error factor of 5\%. This limit is obvious in Figure 7 where the ac conductivity measurements for the dehydrated specimen are conducted in the frequency range between $10 \mathrm{kHz}$ and $1 \mathrm{MHz}$.

The ac conductivity behaviour at the frequency of 10 $\mathrm{kHz}$ was selected to be studied with respect to the applied mechanical load. This frequency was selected since the phenomena of ac conductivity variation due to stress and due to water content become more intense in the low frequency range. Additionally, this frequency is high enough to avoid hardware limitations due to low conductivity effects, thus, ensuring that the total measurement factor will not exceed $1 \%$.

Figure 8 shows the relative ac conductivity for the frequency of $10 \mathrm{kHz}$ with respect to the applied uniaxial load for both dehydrated (a) and hydrated (b) type-1 specimens. The value measured before any load application was considered as reference conductivity. The vertical axis corresponds to the relative conductivity $\left(\sigma / \sigma_{0}\right)$ where $\sigma_{0}$ is the reference conductivity and $\sigma$ is the 10 $\mathrm{kHz}$ conductivity value. The horizontal axis corresponds to the applied load.

The Figure 8 also contains the ac conductivity deviation limits in order to include the results from all measured specimens. The circles in the figure correspond to the mean value of ac conductivity for each value of the applied load.

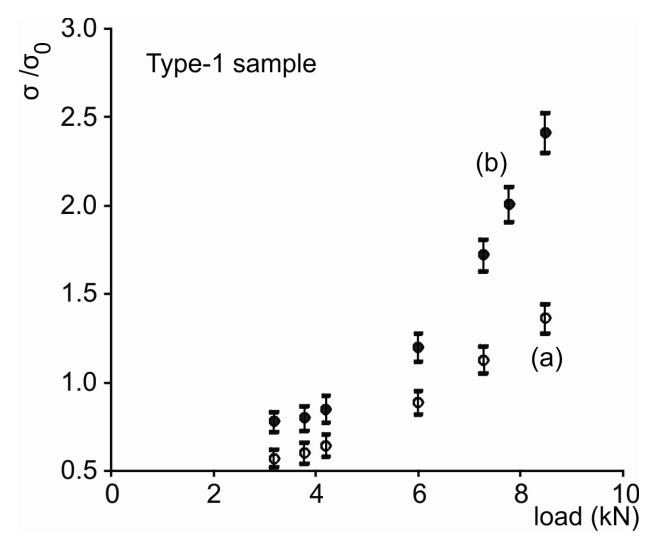

Figure 8. Representative plot of the behaviour of ac conductivity for all type-1 specimens in both before (b) and after (a) removing their natural moisture. 


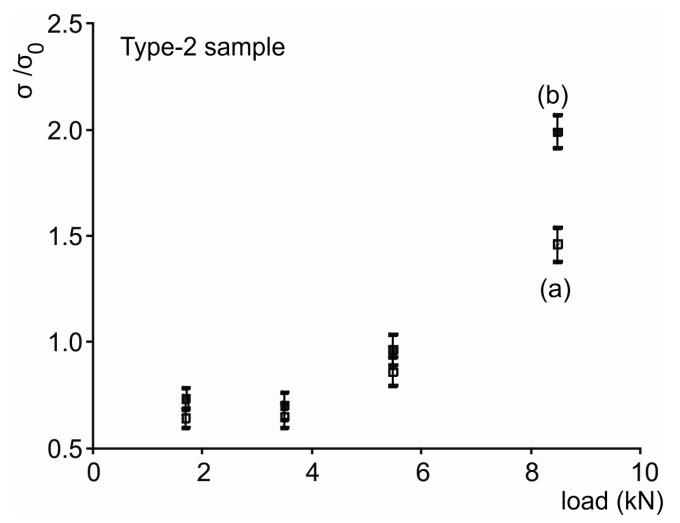

Figure 9. Representative plot of the behaviour of ac conductivity for all type-2 specimens in both before (b) and after (a) removing their natural moisture.

Observing Figure 8 it can be stressed that for type-1 specimens that was subjected to load up to approximately $3 \mathrm{kN}$, the ac conductivity decreases while the applied load increases. This can be attributed to the pore-closing process that takes place in this load range and the consequent limitation of the conductive paths in the bulk of the specimen.

The ac conductivity of the specimens that were subjected to load in the range between $3 \mathrm{kN}$ and $7.5 \mathrm{kN}$, increases significantly with increasing load. This increase can be attributed to the crack propagation mechanisms that create additional conductive paths at the crack edges.

The behaviour of the dehydrated specimens was similar but exhibited lower conductivity magnitudes. The lower magnitude of the changes of the ac conductivity can be attributed to the absence of water in the bulk of the specimen. It can be concluded that ac conductivity measurements can provide information regarding the mechanical status of marble specimens that have suffered stress enough to create damages in their bulk. The same behaviour holds for type 2 specimens and it is shown in Figure 9 with slightly smaller variations.

\section{Conclusions}

The presented experimental results manifest that uniaxial compressional loading, causes significant variations to the ac conductivity spectrum of marble specimens.

The applied uniaxial load when leading to microcrack generation in the bulk of the specimens makes conductivity to increase due to hydration within the microcracks.

For the marble specimens that were subjected to uniaxial compressional load the ac conductivity was measured in the frequency range $1 \mathrm{kHz}$ up to $1 \mathrm{MHz}$. The re- sults presented here correspond to the frequency of 10 $\mathrm{kHz}$ since it is the most sensitive to show up ac conductivity variations for changes of load.

\section{REFERENCES}

[1] F. G. Bell, "Engineering Properties of Soils and Rocks," Blackwell Science Publishers, Hoboken, 2000.

[2] J. C. Jaeger and N. G. W. Cook, "Fundamentals of Rock Mechanics," Chapman and Hall Publishers, London, 1979. doi:10.1017/CBO9780511735349

[3] D. L. Turcotte, W. I. Newman and R. Shcherbakov, "Micro and Macroscopic Models of Rock Fracture," Geophysical Journal International, Vol. 152, No. 3, 2003, pp. 718-728. doi:10.1046/j.1365-246X.2003.01884.x

[4] D. Triantis, C. Anastasiadis, I. Stavrakas and F. Vallianatos, "Dielectric Characteristics of Marble Rocks after the Application of Various Stress Modes before Fracture," WSEAS Transactions on Systems, Vol. 3, 2004, pp. 102106.

[5] C. Anastasiadis, I. Stavrakas, D. Triantis, A. Kyriazopoulos and K. Ninos, "Rock Damage Estimation with Dielectric Loss $(\tan \delta)$ Measurements," International Journal of Microstructure and Materials Properties, Vol. 1, No. 3-4, 2006, pp. 421-429.

[6] I. Stavrakas, D. Triantis and C. Anastasiadis, "The Influence of Externally Applied Uniaxial Stress on Isothermal Depolarization Current Mechanisms in Rock Samples," Journal of Material Science, Vol. 40, No. 17, 2005, pp. 4593-4596. doi:10.1007/s10853-005-1152-3

[7] T. L. Chelidze, Y. Gueguen and C. Ruffet, "Electrical Spectroscopy of Porous Rocks: A Review-II. Experimental Results and Interpretation," Geophysical Journal International, Vol. 137, No. 1, 1999, pp. 16-34. doi:10.1046/j.1365-246x.1999.00800.x

[8] T. L. Chelidze and Y. Gueguen, "Electrical spectroscopy of Porous Rocks: A Review-I. Theoretical Model," Geophysical Journal International, Vol. 137, No. 1, 1999, pp. 1-15. doi:10.1046/j.1365-246x.1999.00799.x

[9] A. Kyritsis, M. Siakantari, A. Vassilikou-Dova, P. Pissis, and P. Varotsos, "Dielectric and Electrical Properties of Polycrystalline Rocks at Various Hydration Levels," IEEE Transactions on Dielectrics and Electrical Insulation, Vol. 7, No. 4, 2000, pp. 493-497.

[10] A. K. Jonscher, "Dielectric Relaxation in Solids," Chelsea Dielectric Press, London, 1983.

[11] J. Dyre, "Some Remarks on ac Conduction in Disordered Solids," Journal of Non-Crystalline Solids, Vol. 135, No. 2-3, 1991, pp. 219-226. doi:10.1016/0022-3093(91)90423-4

[12] C. Anastasiadis, D. Triantis, I. Stavrakas, A. Kyriazopoulos and F. Vallianatos, "Ac Conductivity Measurements of Rock Samples after the Application of Stress Up to Fracture. Correlation with the Damage Variable," WSEAS Transactions on Systems, Vol. 4, No. 3, 2005, pp. 185190. 